\title{
STAT3-activated CD36 facilitates fatty acid uptake in chronic Iymphocytic leukemia cells
}

\author{
Uri Rozovski, ${ }^{1,2}$, David M. Harris ${ }^{1}$, Ping Li $^{1}$, Zhiming Liu ${ }^{1}$, Preetesh Jain ${ }^{1}$, Alessandra \\ Ferrajoli $^{1}$, Jan Burger ${ }^{1}$, Phillip Thompson ${ }^{1}$, Nitin Jain ${ }^{1}$, William Wierda ${ }^{1}$, Michael $\mathrm{J}$. \\ Keating ${ }^{1}$ and Zeev Estrov ${ }^{1}$ \\ ${ }^{1}$ Department of Leukemia, The University of Texas MD Anderson Cancer Center, Houston, Texas, USA \\ ${ }^{2}$ Institute of Hematology, Davidoff Cancer Center, Rabin Medical Center, Sackler School of Medicine, Tel Aviv University, Tel \\ Aviv, Israel
}

Correspondence to: Zeev Estrov, email: zestrov@mdanderson.org

Keywords: CLL; CD36; metabolism; STAT3

Received: January 31, $2018 \quad$ Accepted: March 21, $2018 \quad$ Published: April 20, 2018

Copyright: Rozovski et al. This is an open-access article distributed under the terms of the Creative Commons Attribution License 3.0 (CC BY 3.0), which permits unrestricted use, distribution, and reproduction in any medium, provided the original author and source are credited.

\section{ABSTRACT}

\begin{abstract}
Although several studies established that unlike normal B cells chronic lymphocytic leukemia (CLL) cells metabolize fatty acids (FA), how CLL cells internalize FA is poorly understood. Because in various cell types CD36 facilitates FA uptake, we wondered whether a similar mechanism is operative CLL. We found that CD36 levels are higher in CLL cells than in normal B cells, and that small interfering RNA, CD36 neutralizing antibodies or sulfosuccinimidyl oleate (SSO) that inhibits CD36 significantly reduced the oxygen consumption of CLL cells incubated with FA. Because CD36 is oeverexpressed and STAT3 is constitutively activated in CLL cells, we wondered whether STAT3 induces CD36 expression. Sequence analysis identified putative STAT3 binding sites in the CD36 gene promoter. Chromatin immunoprecipitation and an electrophoretic mobility shift assay revealed that STAT3 binds to the CD36 gene promoter. A luciferase assay and STAT3-small hairpin RNA, that significantly decreased the levels of CD36 in CLL cells, established that STAT3 activates the transcription of the CD36 gene. Furthermore, SSO induced a dose-dependent apoptosis of CLL cells. Taken together, our data suggest that STAT3 activates CD36 and that CD36 facilitates FA uptake in CLL cells. Whether CD36 inhibition would provide clinical benefits in CLL remains to be determined.
\end{abstract}

\section{INTRODUCTION}

Chronic lymphocytic leukemia (CLL) is characterized by a gradual increase in the number of circulating mature appearing lymphocytes. For more than four decades CLL was viewed as a disease characterized by an accumulation of long-lived mature looking neoplastic B-lymphocytes that do not proliferate and do not die. Studies conducted in recent years clearly demonstrated that CLL cells do proliferate [1-3] and undergo spontaneous apoptosis [4]. The proliferation rate of CLL cells varies, however based on heavy water labeling studies it is estimated that approximately $1 \%$ of the CLL clone expands daily [5], an expansion rate that is remarkably similar to that of normal adipocytes [6].

We and others $[7,8]$ have recently shown that, unlike normal B cells, CLL cells adopt metabolic pathways that are operative in adipocytes and myocytes. Specifically, we found that CLL cells store lipids in intracytoplasmic vacuoles and, by aberrantly expressing lipoprotein lipase (LPL), utilize fatty acids (FA) for the production of chemical energy $[8,9]$. We have also shown that LPL expression and FA metabolism in CLL is driven by constitutive activation of the signal transducer and 
activator of transcription (STAT)-3. However, what is the source of FA and what mechanisms are recruited to enable FA entry into CLL cells is not completely understood.

CD36, also known as fatty acid translocase protein, is a multi-ligand glycoprotein that is expressed on the extracellular membrane and facilitates FA uptake in various cells such as myocytes and adipocytes [10] in which lipid uptake is markedly impaired when CD36 function is compromised [11-13]. Because CLL cells' metabolic pathways resembles those of adipocytes [14] and the CD36 gene harbors putative STAT3-binding sites, we hypothesized that STAT3 induces CD36 cell surface expression and that CD36 facilitates FA uptake in CLL cells.

\section{RESULTS}

\section{CLL cells express high CD36 protein levels of}

To assess the levels of CD36 protein in CLL cells we performed Western blot analysis of PB-derived CLL cells from 6 randomly chosen CLL patients and, as control, CD19+ B cells from the PB of two healthy individuals. As shown in Figure 1A, protein levels of CD36 were readily detected in all CLL-patient samples and their levels were significantly higher than those of CD19+ B cells obtained from healthy individuals. As in previous studies [15], phosphoserine STAT3 was detected in CLL cells but its levels were undetectable in normal CD19+ B cells. To confirm that CLL cells express CD36 cell membrane protein we performed flow cytometry and detected CD36 protein on the surface of PB CLL cells from 6 randomly selected CLL patients (Figure 1B). However, the percentages of cells co-expressing CD19, CD5, and CD36 antigens varied among the different patients and were 14, $25,32.9,37,41$, and 51, respectively. To confirm these findings we used confocal microscopy and found that, like in other cell types, CD36 is detected on the surface but not in the cytoplasm or nucleus of CLL cells (Figure 1C).

\section{STAT3 binds to the CD36 gene promoter and activates the CD36 gene}

Because STAT3 is constitutively activated in CLL cells [15], we wondered whether overexpression of CD36 is driven by STAT3. Using the TFSEARCH database [16] we identified 4 GAS-like elements, known as putative STAT3-binding sites [17], within $600 \mathrm{bp}$ upstream of the CD36 gene start codon (Figure 2A). To determine whether STAT3 binds to any of these GAS-like elements we performed ChIP. Our ChIP analysis revealed that 3 DNA fragments, whose primers amplified the regions of the putative STAT3 binding sites $+187 \mathrm{bp}-+196 \mathrm{bp}$, $+363 \mathrm{bp}-+374 \mathrm{bp}$, and $+382 \mathrm{bp}-+391 \mathrm{bp}$, but not the region of the putative STAT3 binding site -93 bp --83 bp, co-immunoprecipitated with anti-STAT3 antibodies
(Figure 2B). To confirm that these DNA fragments bind STAT3 we performed EMSA using biotinylated DNA probes corresponding to the binding sites $+187 \mathrm{bp}-+196$ bp, $+363 \mathrm{bp}-+374 \mathrm{bp}$, and $+382 \mathrm{bp}-+391 \mathrm{bp}$, and -93 bp --83 bp. Confirming the ChIP data, we found that CLL cell nuclear protein extracts from 2 different patients formed complexes with STAT3 binding sites $+187 \mathrm{bp}-$ $+196 \mathrm{bp},+363 \mathrm{bp}-+374 \mathrm{bp}$, and $+382 \mathrm{bp}-+391 \mathrm{bp}$, but not with the putative binding site $-93 \mathrm{bp}--83 \mathrm{bp}$ and that the binding was significantly attenuated by the addition of excess unlabeled probe or by anti-STAT3 antibodies (Figure 2C). To further delineate these findings we performed a luciferase assay of MM1 cells in which we concentrated on putative STAT3 binding sites that were in close proximity to the CD36 gene start codon. We found the highest luciferase activity in IL-6 stimulated MM1 cells transfected with the promoter fragment that included the 3 active binding sites (Figure 2D). Furthermore, we found that STAT3-shRNA downregulated mRNA levels both of STAT3 and CD36 by 9 and 6-fold, respectively, and as a result, significantly reduced STAT3 and CD36 protein levels, confirming that STAT3 induces the expression of CD36 in CLL cells (Figure 2E).

\section{CD36 facilitates FA intake and metabolism in CLL cells}

Because CLL cells utilize FA and CD36 plays a key role in FA uptake in various cell types $[18,19]$, we sought to determine whether CD36 contributes to FA uptake in CLL cells. We cultured CLL cells in tightly closed flaks in serum- and glucose-free medium and measured the oxygen concentration prior to and after adding FA, assuming that if the cells consume the FA, oxygen levels in the culture medium will drop. As expected, when FA were added to culture the levels of oxygen dissolved in the culture media were markedly reduced whereas the $\mathrm{dO}_{2}$ levels of CLL cells transfected with CD36 siRNA and incubated with oleic acid, remained significantly higher than the $\mathrm{dO}_{2}$ levels in the medium of non-transfected or GPDH-transfected CLL cells (Figure $3 \mathrm{~A}$ ). Similarly, the $\mathrm{dO}_{2}$ levels of CLL cells incubated with oleic acid or palmitic acid in the absence of CD36 neutralizing antibodies significantly dropped whereas $\mathrm{dO}_{2}$ levels of CLL cells incubated with oleic acid or palmitic acid in the presence of CD36 neutralizing antibodies remained unchanged (Figure 3B). Likewise, the irreversible CD36 inhibitor SSO and the LPL inhibitor orlistat significantly reduced CLL cell $\mathrm{O}_{2}$ consumption and the effect of both inhibitors was significantly additive (Figure 3C). Furthermore, SSO induced apoptosis of CLL cells in a dose-dependent manner (Figure 3D).

\section{DISCUSSION}

Here we show that CLL cells express high levels of CD36 cell surface protein, that overexpression of CD36 is 
driven by STAT3-mediated activation of the CD36 gene, and that CD36 facilitates uptake of FA by CLL cells.

Although CLL is a heterogeneous disease, in all patients, regardless of clinical characteristics, disease burden, cytogenetic abnormalities or IgHv gene mutation status, STAT3 is constitutively phosphorylated on serine 727 residues $[15,20]$. Phosphoserine STAT3 activates a diverse repertoire of coding and non-coding genes which protect CLL cells from apoptosis and provide the cells with proliferation capacity [8, 21-23]. The common constitutive activation of STAT3 in all CLL patients suggests that STAT3 is involved in a core function that affects CLL cell survival.

Several observations linked STAT3 to cellular metabolism in malignant and non-malignant cells [24]. In cancer cells STAT3 emerged as a as a key regulator of metabolism that integrates signals via both mitochondrial and nuclear activities [25]. We have recently shown that

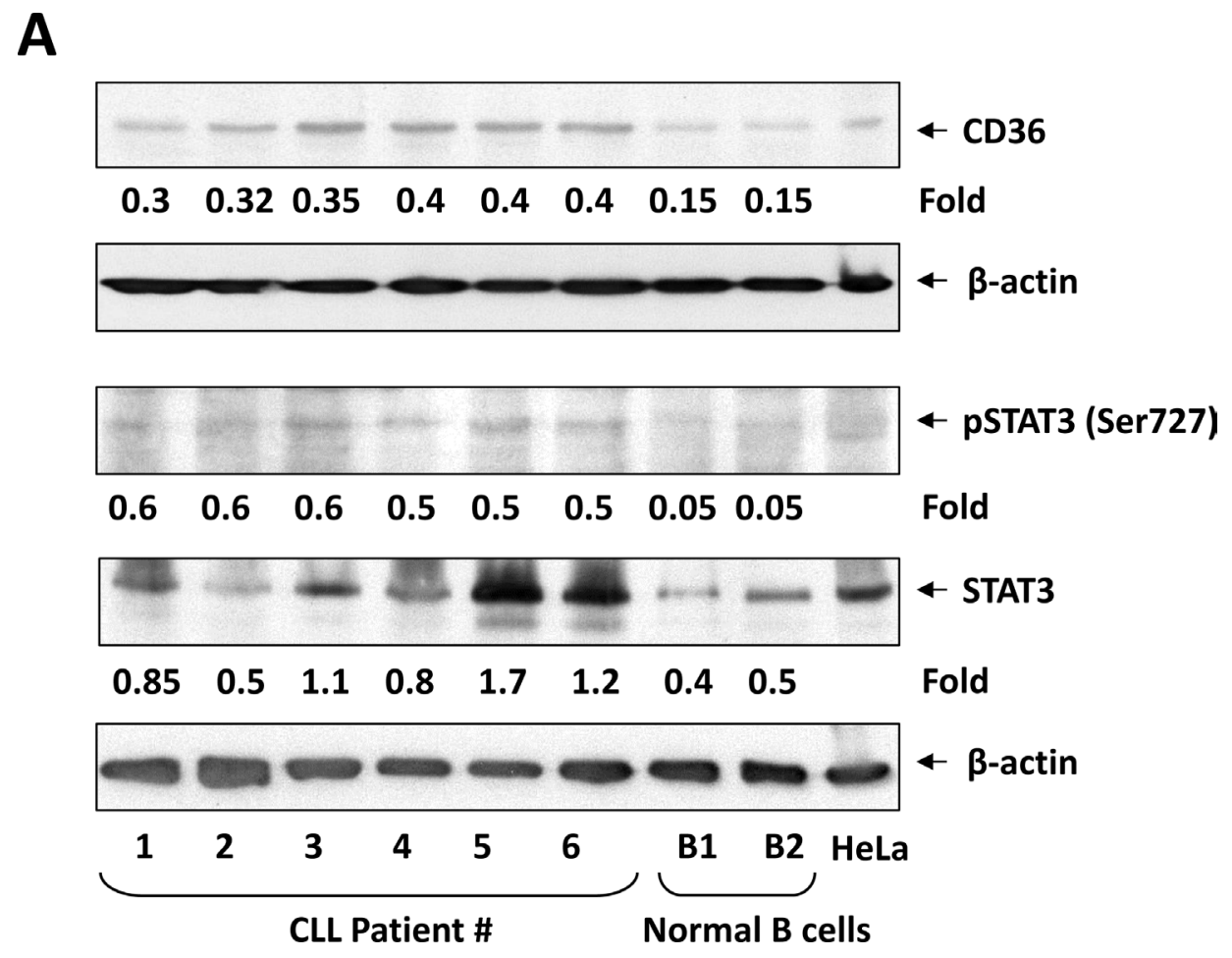

B
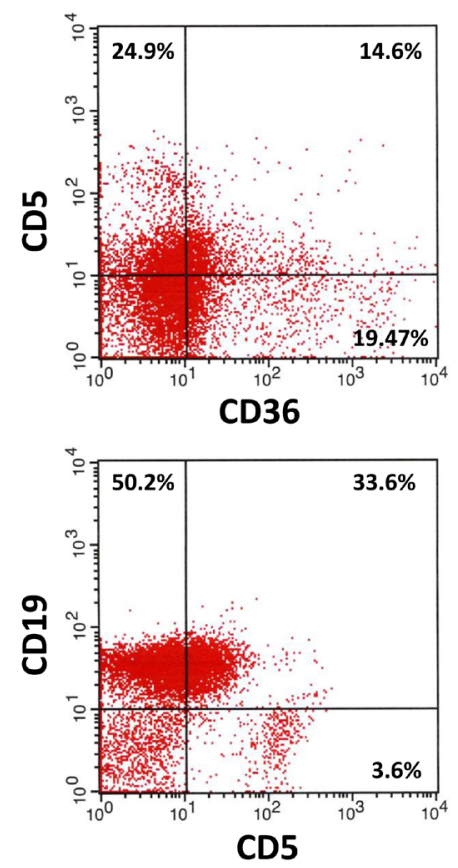
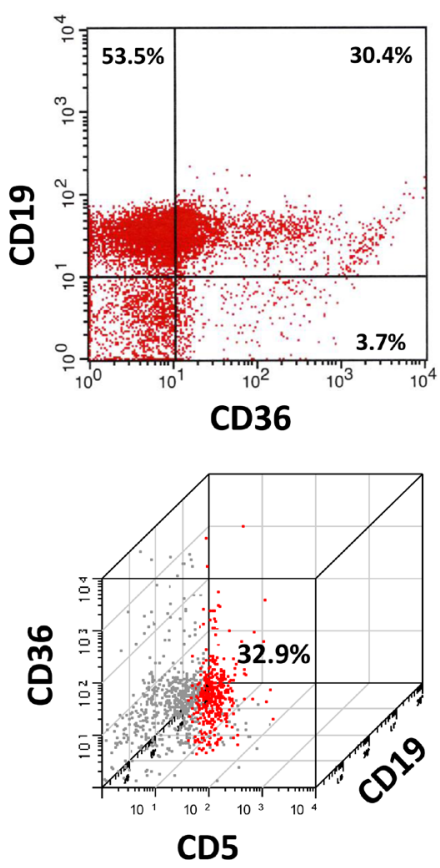

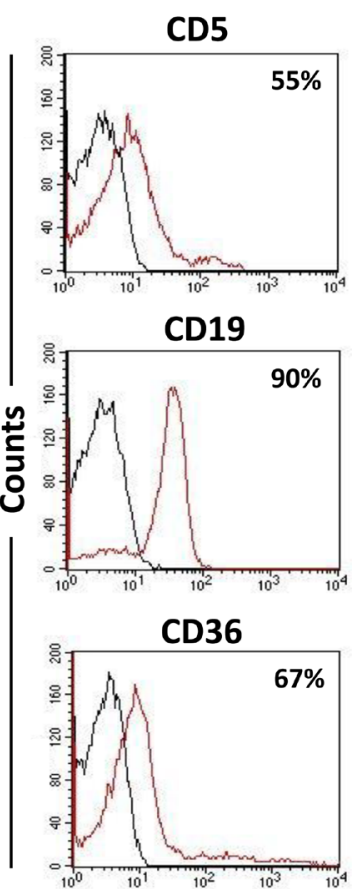




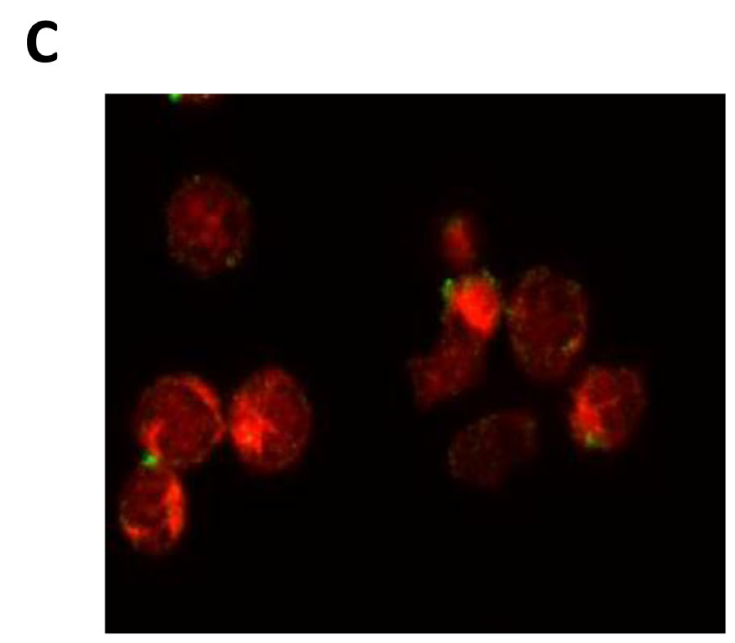

Normal B Cells

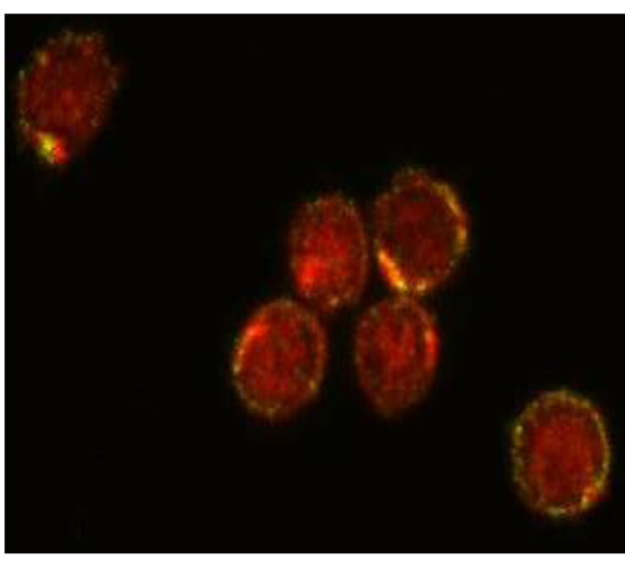

CLL Cells

Figure 1: CLL cells express high levels of CD36. (A) Western blot analysis of CLL cells from 6 different patients and CD19+ normal B cells from 2 normal controls. In all patients samples CD36 was detected at higher levels than in normal B cells. Phosphoserine STAT3 was detected in all patient samples but not in CD19+ normal B cells and levels of unphosphorylated STAT3 were higher in CLL patient samples than in CD19+ normal B cells. Actin was used as loading control and densitometry units were normalized to actin units in the corresponding lanes. (B) CLL cells co-express CD19, CD5 and CD36. Left panel: A representative figure of flow cytometry analysis performed on CLL cells from 6 different patients is depicted. Right panel: Isotype controls. The black line is Isotype control and the red line is the corresponding antibody. Percentages represent the differences between the isotype and the specific antibody. The precentages are the (C) Confocal microscopic images $(\times 400)$ of freshly isolated CLL cells (right) and normal B cells (left) stained with anti-CD36 antibodies (green) and Evans blue (red) showing CD36 on the cell surface of CLL cells but not on normal B cells.

by inducing LPL production, STAT3 promotes uptake and metabolism of lipid particles [8]. In various cell types including CLL cells, lipid particles are stored in vacuoles and following additional processing triglycerides undergo hydrolysis into FA generating substrates that enter the Krebs cycle. Here we show that CLL cells utilize a complementary strategy for a direct uptake of circulating FA by STAT3-mediated expression of CD36.

Studies in CD36-null mice identified CD36 as a facilitator of FA uptake [26]. In addition, several groups reported that CD36-mediated FA uptake plays a role in the pathogenesis of various neoplasms. For example, Nath et al. showed that in hepatocellular carcinoma CD36mediated uptake of FA induces epithelial to mesenchymal transition and metastasis [27]. Likewise, CD36-mediated
FA uptake was found to promote metastatic development in a subpopulation of human oral carcinoma cells that express high levels of CD36 [28].

To evaluate the role of CD36 in CLL cells we used three different strategies. We downregulated CD36 mRNA levels by transfecting CLL cells with CD36 siRNA and, in other experiments, blocked CD36 activity with CD36neutralizing antibodies or SSO. Using these three different methods we consistently found that inhibition of CD36 disrupted FA metabolism, confirming that in CLL cells CD36 is required for FA uptake.

Sulfo-N-succinimidyl oleate (SSO) attaches to the CD36 binding pocket and, by doing so, it inhibits CD36-mediated FA uptake [29]. Therefore SSO has been extensively used to study the role of CD36. For example,

A

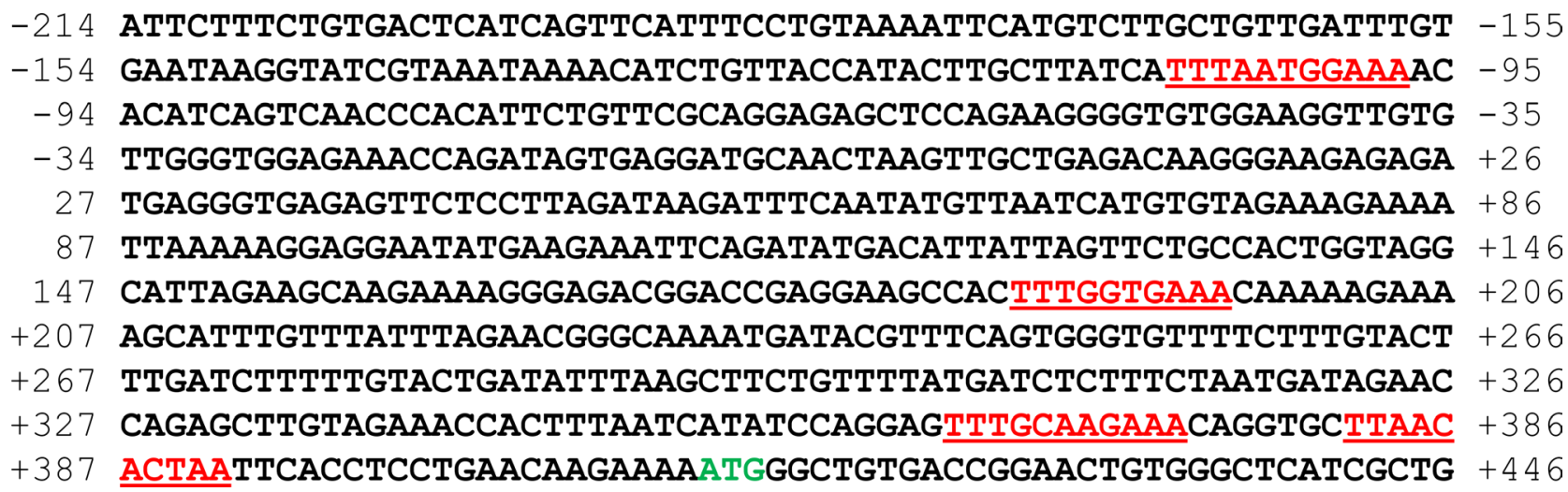


B

Input STAT3 IgG

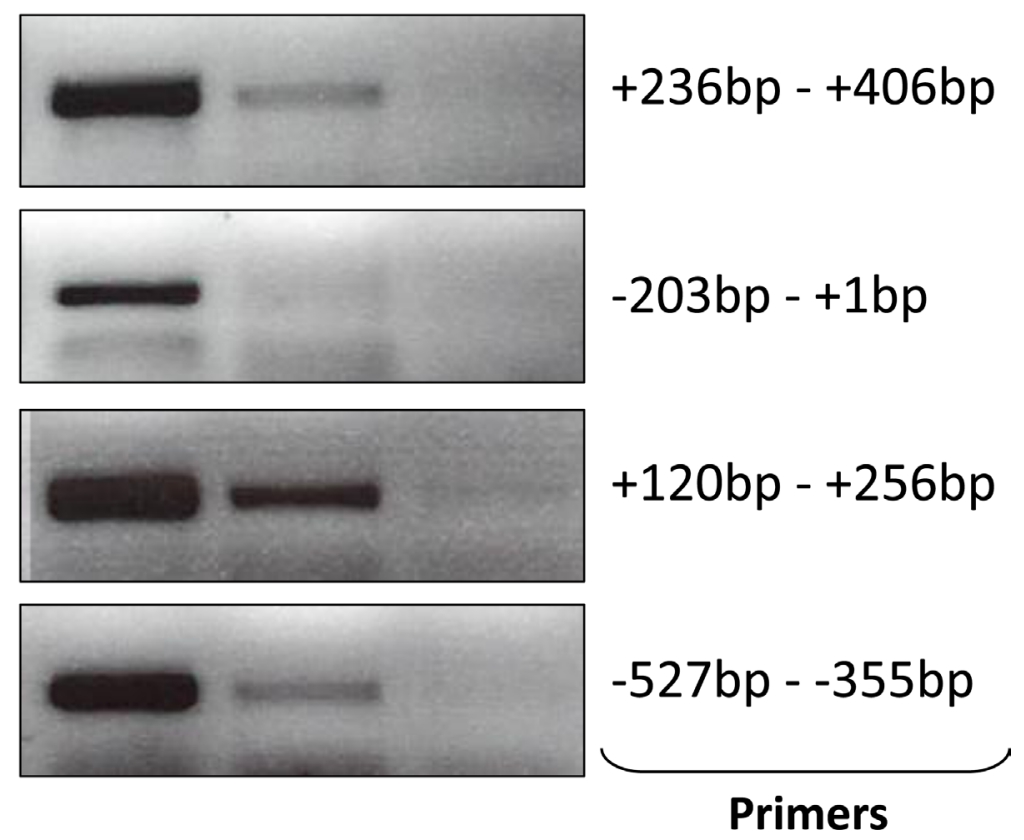

C

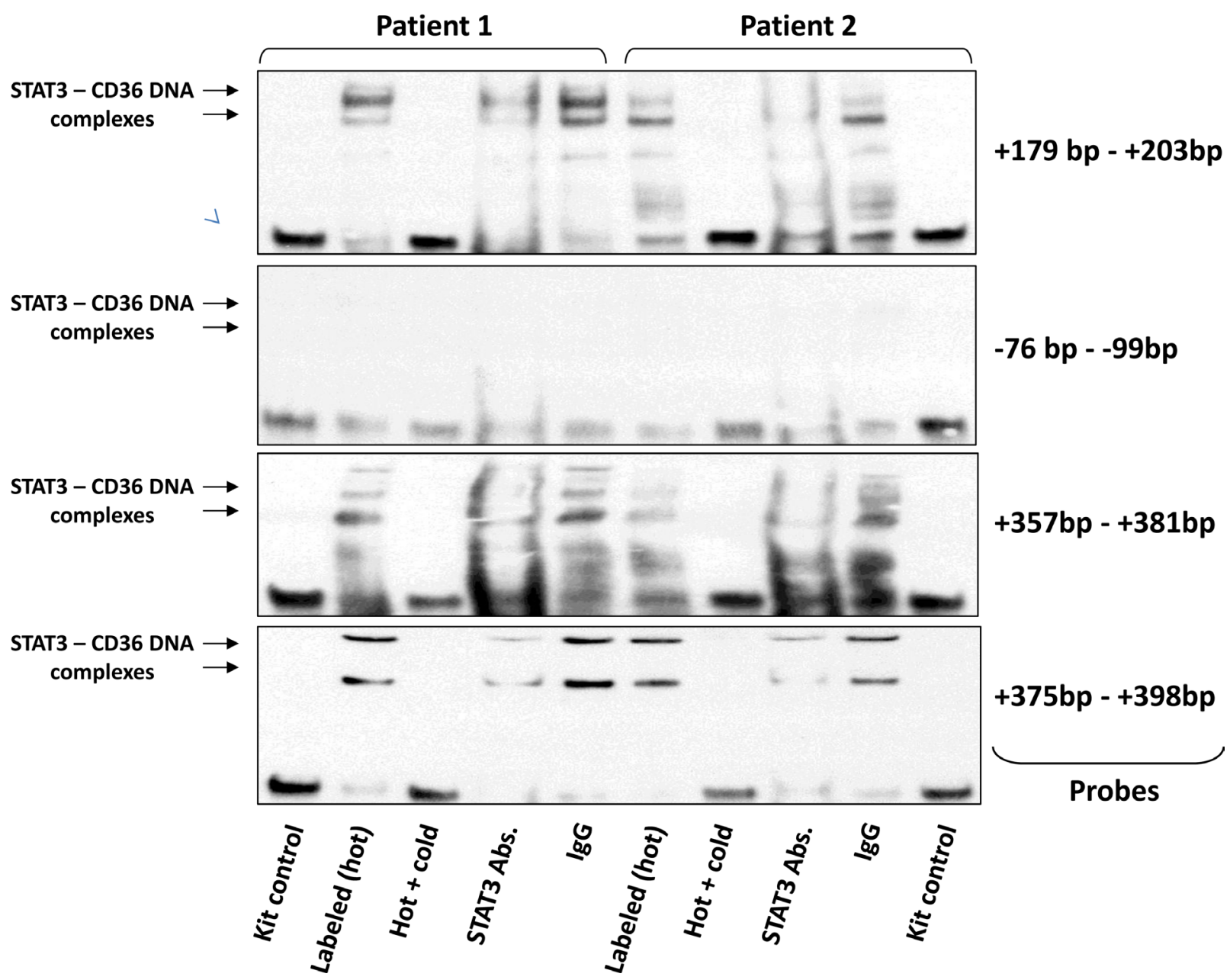




\section{D}
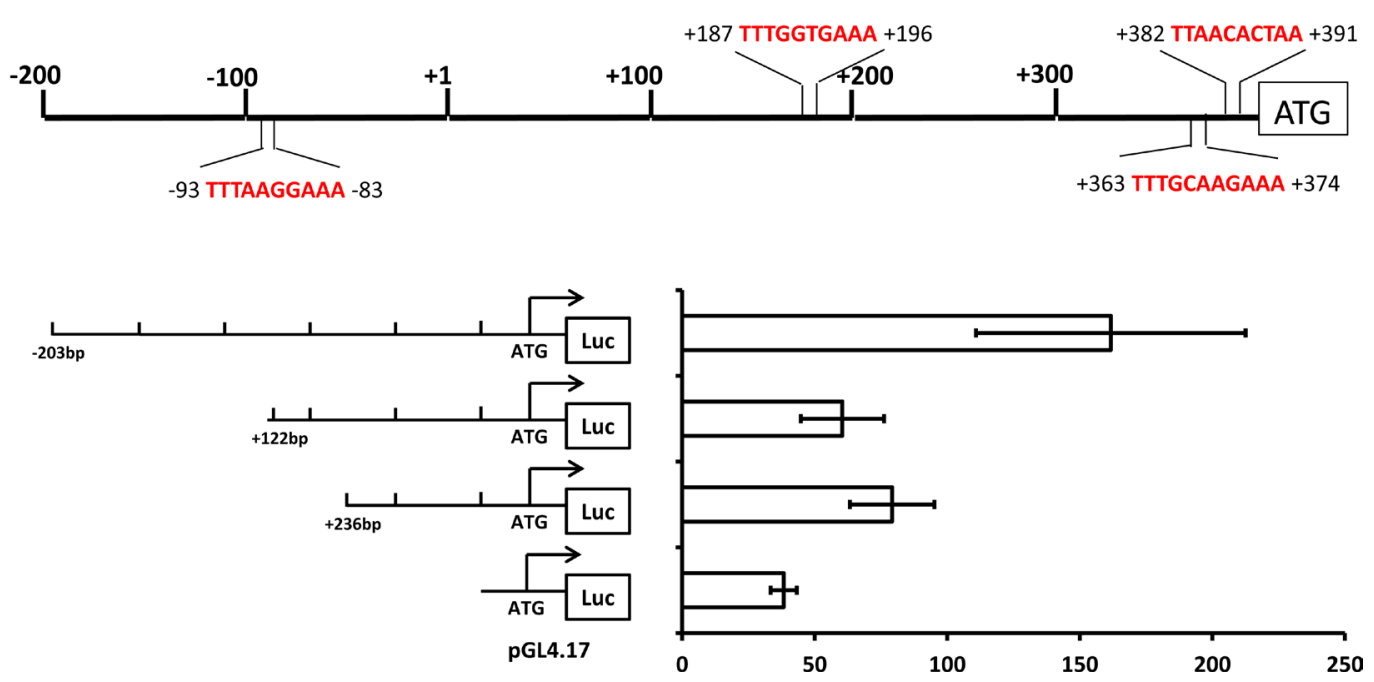

E
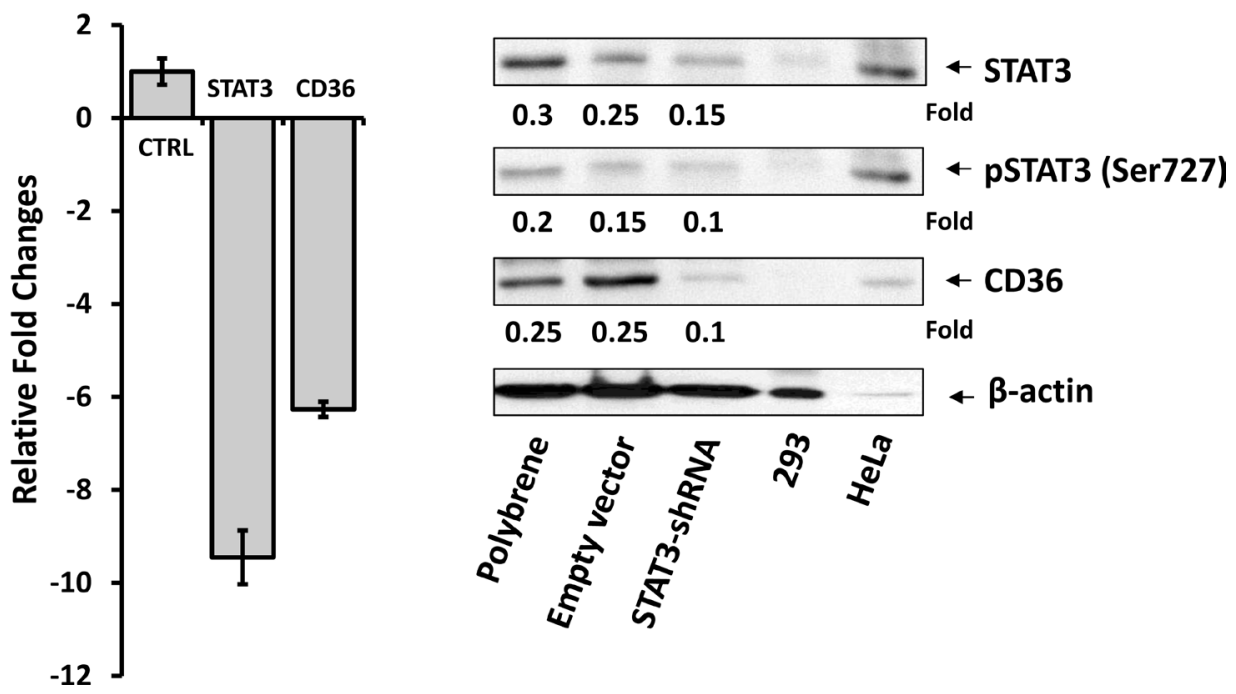

Figure 2: STAT3 binds to and activates the CD36 gene promoter. (A) Sequence analysis of the CD36 gene promoter upstream of the start codon (green) revealed 4 putative STAT3 binding sites (red). Upstream putative STAT3 biding sites are not shown because in most experiments we concentrated our efforts on studying binding sites that are in proximity to the CD36 start codon. (B) Using the ChIP method, CLL-cell chromatin fragments pulled down by anti-STAT3 antibodies were analyzed by PCR using primers directed at the 4 putative STAT3 binding sites upstream the CD36 gene start codon. As shown, anti-STAT3 antibodies co-immunoprecipitated the DNA detected by primers $+236 \mathrm{bp}-+406 \mathrm{bp},+120 \mathrm{bp}-+256 \mathrm{bp}$ (amplifying regions of the STAT3 putative binding sites $+187 \mathrm{bp}-+196 \mathrm{bp}$, $+363 \mathrm{bp}-374 \mathrm{bp}$, and $+382 \mathrm{bp}-+391 \mathrm{bp}$ ), and $-527 \mathrm{bp}--355 \mathrm{bp}$ but not by primers $-203 \mathrm{bp}-+1 \mathrm{bp}$ (amplifying the region of the putative binding site -93 bp - $83 \mathrm{bp}$ ). (C) Using EMSA, biotin-labeled CD36-DNA probes were incubated with CLL cells' protein extract from 2 patients. The EMSA demonstrated that CLL cell nuclear protein extracts bound to the CD36 gene promoter at regions that include the putative STAT3 binding sites $+187 \mathrm{bp}-+196 \mathrm{bp},+363 \mathrm{bp}-+374 \mathrm{bp}$, and $+382 \mathrm{bp}-+391 \mathrm{bp}$, but not the region that includes the putative binding site $-93 \mathrm{bp}-83 \mathrm{bp}$, and that the addition of excess unlabeled probe or anti-STAT3 antibodies attenuated the binding. (D) The luciferase activity of IL-6-stimulated MM-1 cells was assessed 24 hours after transfection with the 3 depicted DNA fragments containing putative STAT3 binding sites in close proximity to the start codon is shown. The luciferase activity of each of the human CD36 promoter constructs was determined by calculating the constructs' luciferase activity relative to the activity of the Renilla luciferase produced by the pRL-SV40 control vector. The luciferase activity of unstimulated MM1 cells (not shown) was similar to that of the pRL-SV40 control vector. The highest luciferase activity, compared to the pGL4.17 (control), in IL-6-stimulated MM1 cells transfected with the promoter fragment that included the 3 active binding sites $(-203 \mathrm{bp} ; P=0.0002)$. A lower albeit increased activity was observed in cells transfected with the promoter fragment that included 2 active binding sites ( $+236 \mathrm{bp} ; P=0.009)$. There was no significant difference in the luciferase activity of fragments $+122 \mathrm{bp}$ and $+236 \mathrm{bp}(P=0.12)$. (E) Infection of CLL cells with STAT3-shRNA, downregulated mRNA levels both of STAT3 and CD36 by 9 and 6 fold, respectively (left panel), and significantly reduced protein levels of STAT3, phsophoserine STAT3 and CD36 (right panel). The figure depicts representative results of 3 different experiments. 
A
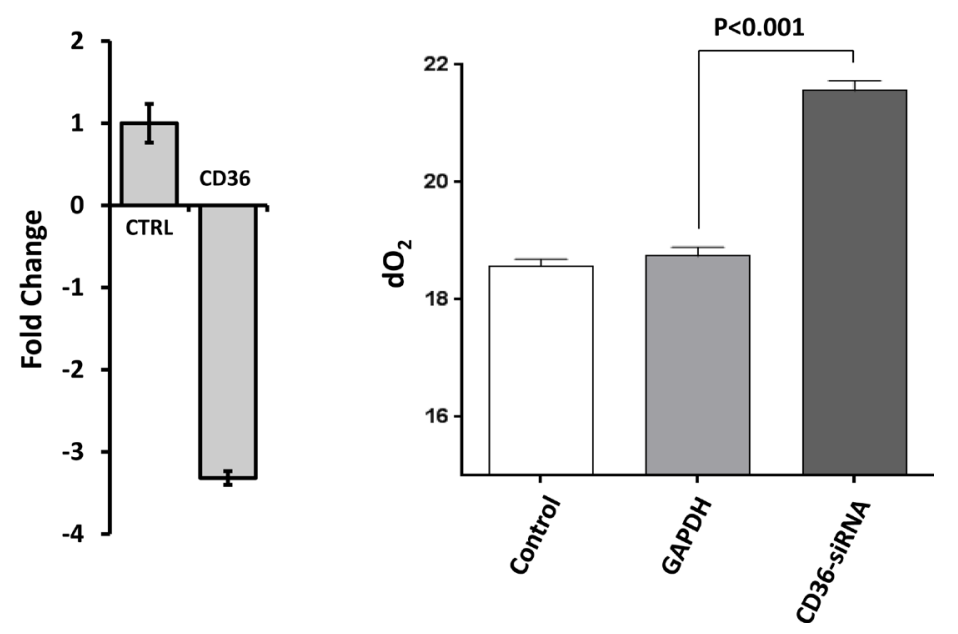

B

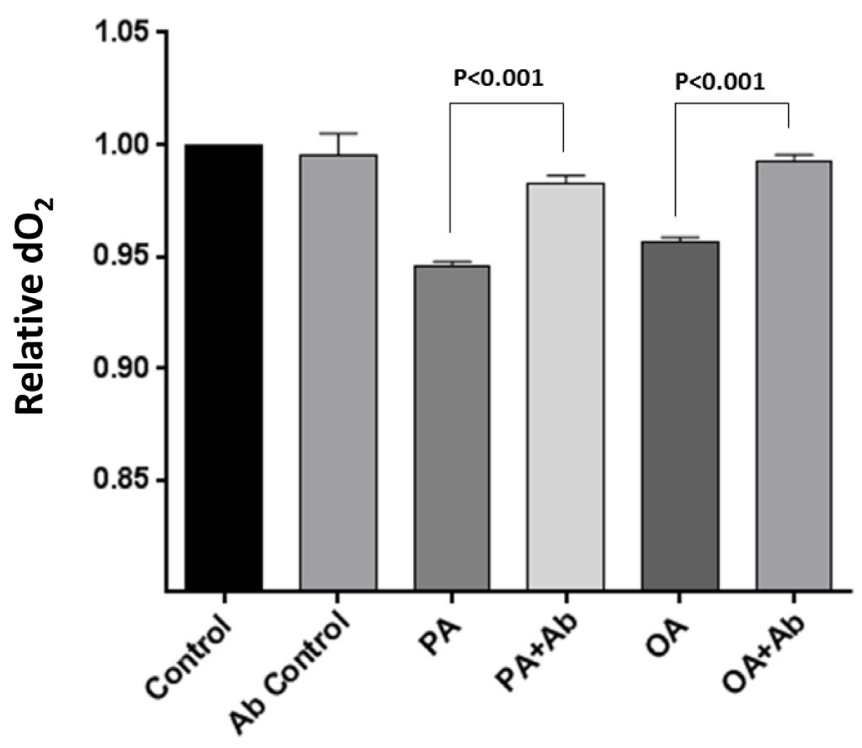

C

Patient 1

Patient 2
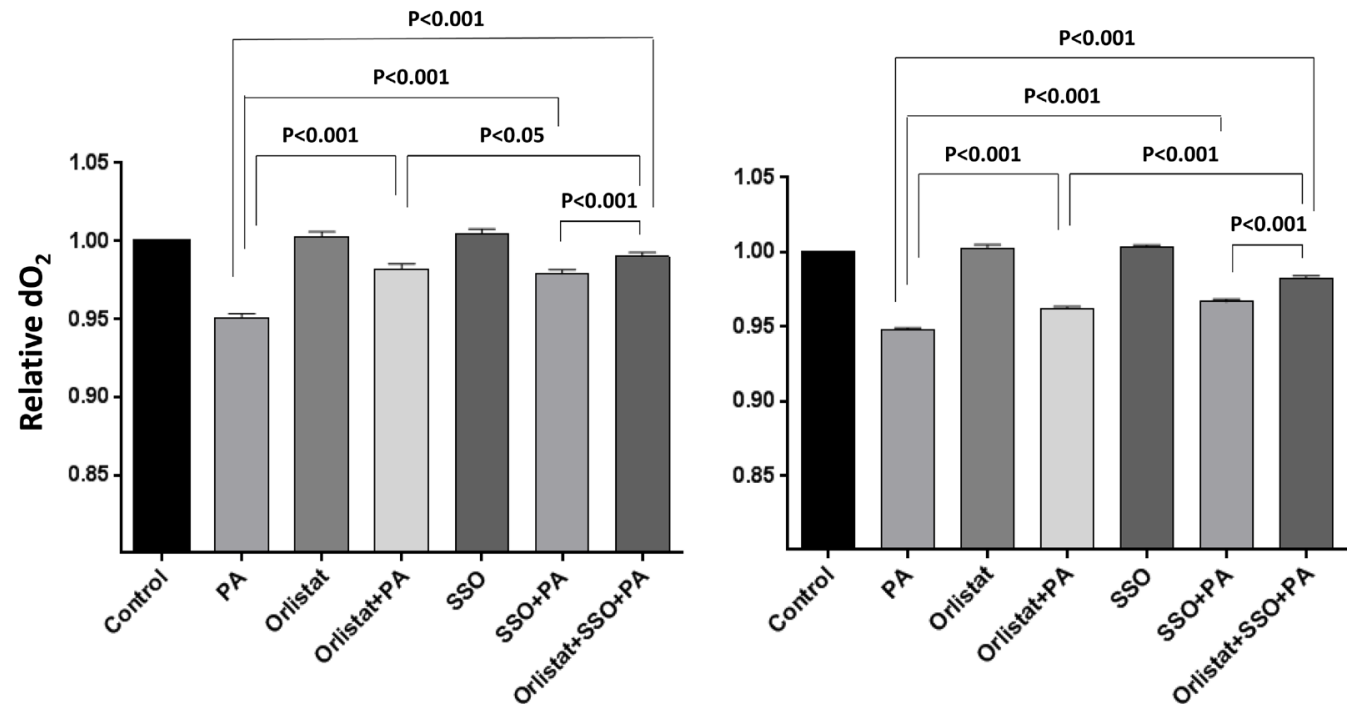


\section{D}
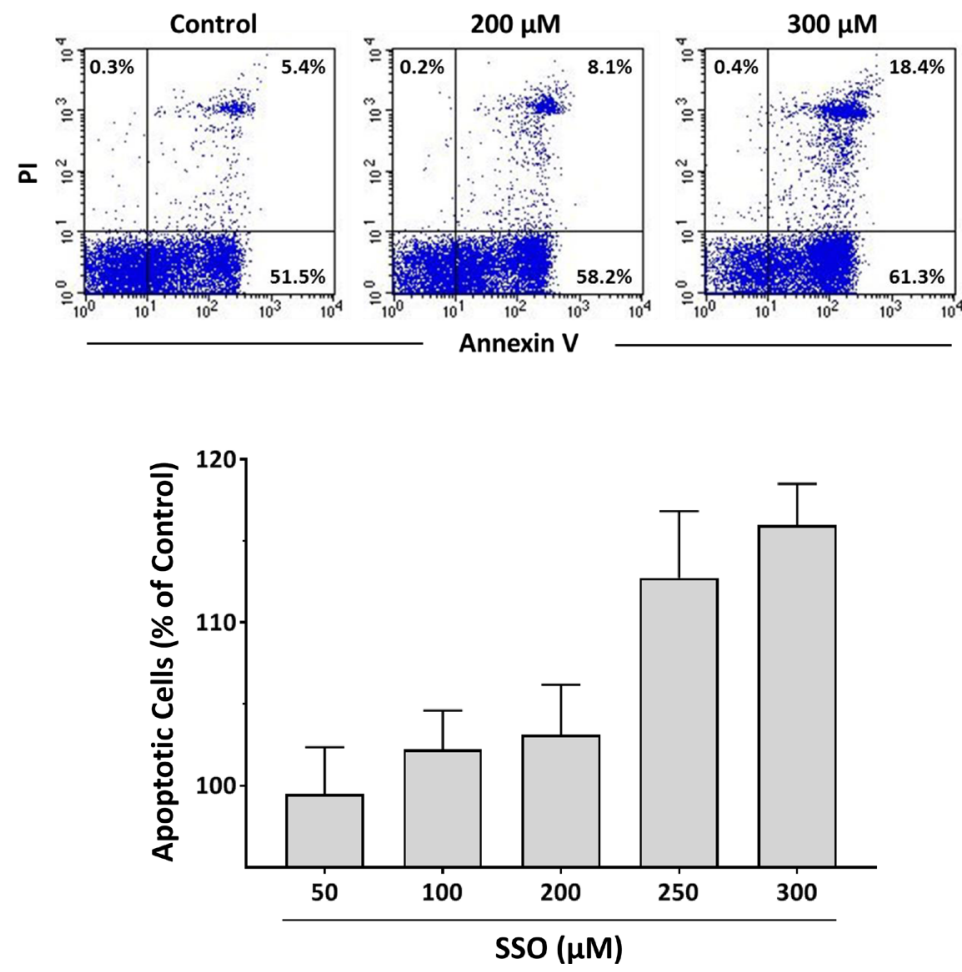

Figure 3: CLL cell FA intake and metabolism is CD36-dependent. (A) CLL cells were transfected with CD36-siRNA or GAPDH and incubated with $80 \mathrm{mM}$ oleic acid in a serum-free, glucose-free medium in tightly sealed flask for 48 hours. Transfection (at a transfection efficiency of $30 \%$ as assessed by flow cytometry; left panel) with CD36-siRNA, but not GAPDH, significantly reduces CD36 mRNA levels compared to transfection with GAPD (CTRL), and the $\mathrm{dO}_{2}$ levels were significantly reduced in the medium of nontransfected or GAPDH-transfected CLL cells but not in the medium of CD36-siRNA-transfected cells (right panel). Representative results from 3 different experiments are depicted. (B) CD36 neutralizing antibodies inhibited FA metabolism in CLL cells. CLL cells from two patients were incubated in the presence or absence of $80 \mathrm{mM}$ palmitic acid (PA) or oleic acid (OA) with or without CD36 neutralizing antibodies $(\mathrm{Ab})$ in sealed tissue culture flasks. The relative $\mathrm{dO}_{2}$ in the culture media was assessed prior to and after 48 hours of incubation. All control cultures contained ethanol at the same concentration as in palmitic acid and oleic acid. To compare $\mathrm{dO}_{2}$ we assessed the difference in $\mathrm{O}_{2}$ concentration before and after the incubation. The relative difference in $\mathrm{dO}_{2}$ before after adding FA is depicted after the $\mathrm{dO}_{2}$ in the controls were set to 1 . As shown, the CD36-neutralizing antibodies did not affect the cells' oxygen consumption whereas in the presence of PA or OA the cells consumed $\mathrm{O}_{2}$, and this effect was reversed when neutralizing antibodies were added to culture. (C) CLL cells from 2 patients were incubated for 48 hours in the presence or absence of $80 \mathrm{mM}$ palmitic acid (PA) with or without the LPL inhibitor orlistat, the CD36 inhibitor SSO or both. The culture media concentration was assessed prior to and after 48 hours of incubation. As shown in the presence of $\mathrm{PA} \mathrm{dO}_{2}$ was reduced, but less when SSO or orlistat were added to culture and the effect of SSO and orlistat was additive, indicating that these agents reduced FA-dependent metabolism. (D) Apoptosis was assessed in CLL cells incubated with or without increasing concentrations of SSO using flow cytometry with annexin V/PI staining. Upper panel: Representative flow cytometry plots of the below depicted three experiments. Lower panel: Results of 3 different experiments, using cells from 3 different patients are depicted as percent of the apoptosis rate in cells incubated without SSO (control).

SSO was found to effectively block CD36-mediated fatty acid uptake into cardiomyocytes [11, 30, 31] and to restore of diabetic heart's function following hypoxia/ reoxygenation [32]. However, SSO is chemically instable and therefore cannot be used in its current form for longterm experiments or in vivo studies [11]. The use of CD36 neutralizing antibodies is an attractive alternative that has been used in animal models $[31,33]$. CD36 neutralizing antibodies were shown to eliminate lymph node metastasis in mice that were inoculated with tumor cells [28], suggesting that it may also be applicable in treatment of CLL-affected lymph nodes.
In conclusion, unlike their normal resting $\mathrm{B}$ cell counterparts, CLL cells utilize FA. FA uptake in CLL cells is facilitated by STAT3-enhanced CD36 expression. Whether inhibition of CD36-dependent FA uptake might have therapeutic benefit in CLL remains to be determined.

\section{MATERIALS AND METHODS}

\section{Patients' characteristics}

Peripheral blood samples were obtained from 19 patients with CLL who were treated at The University of 
Texas MD Anderson Cancer Center Clinic. The study was approved by our Institutional Review Board and patients' Informed Consent was obtained prior to sample collection. Clinical characteristics of all patients that participated in this study are depicted in Table 1.

\section{Cell fractionation}

PB cells were fractionated using Ficoll Hypaque 1077 (Sigma, St. Louis, MO). The low-density cellular fraction was used immediately or frozen for additional studies. More than $95 \%$ of the peripheral blood lymphocytes obtained from these patients were CD19+/CD5+, as assessed by flow cytometry (Becton, Dickinson and Company, Franklin Lakes, NJ). As control studies we obtained from the Central Blood Bank leftover buffy coats of healthy blood donors. After FicollHypaque fractionation, the donors' B cells were isolated using Miltenyi CD19-coated beads according to the manufacturer's instructions (Miltenyi Biotec, Bergisch Gladbach, Germany).

\section{Western immunoblotting}

Western immunoblotting was performed as previously described [34]. Briefly, CLL cell extract was prepared. The protein concentration was determined using a Micro BCA protein assay reagent kit (Thermo Scientific, Pierce, Rockford, IL). Cell lysates were denatured and following electrophoresis transferred to a nitrocellulose membranes. The membranes were incubated with either monoclonal mouse anti-human STAT3 (BD Bioscience, Palo Alto, CA), polyclonal rabbit anti-human phosphoserine (serine 727) STAT3 (Cell Signaling Technology, Beverly, MA), or rabbit anti-human CD36 (Pierce, Thermo Scientific, Waltham, MA) antibodies and Horseradish peroxidase-conjugated secondary antibodies (GE Healthcare, Amersham, Buckinghamshire, UK). Proteins were visualized via an enhanced chemiluminescence detection system (GE Healthcare).

\section{Flow cytometry analysis}

CLL cells were fixed in $2 \%$ paraformaldehyde for 10 minutes and permeabilized overnight at $-20^{\circ} \mathrm{C}$. Before staining cells were washed three times in PBS with $2 \%$ FBS. Cells were then stained with CD19 (BD Biosciences, San Jose CA), CD5 (BD Biosciences), and CD36 (BD Biosciences) as well as appropriate isotypic controls. Cells were analyzed on a FacsCaliber flow cytometer (BD Biosciences) and data analysis performed using CellQuest software (BD Biosciences). Graphics were created with CellQuest (BD Biosciences) and WinList (Verity Software House, Topsham, ME) software.

\section{Confocal microscopy}

A previously described method was used [35]. CLL low-density cells were incubated in microtubes in PBS supplemented with 5\% bovine serum albumin (Cell Signaling Technology,). After 1 hour of incubation, the cells were washed three times with PBS and then incubated with mouse anti-CD36 antibodies (Novus Scientific, Littleton, CO) for 1 hour, washed in PBS, resuspended in a $0.1 \%$ solution of Evans blue dye (SigmaAldrich) for 5 minutes, and washed in PBS to remove unbound dye. The cells were resuspended in PBS and placed into $\mu$-slide $\mathrm{VI}^{0.4}$ chamber slides (ibidi LLC, Verona, WI) for microscopic analysis. The slides were viewed using an Olympus FluoView 500 Laser Scanning Confocal Microscope (Olympus America, Center Valley, PA), and images were analyzed using the FluoView software (Olympus America).

\section{Chromatin immunoprecipitation (ChIP) assay}

A chromatin immunoprecipitation (ChIP) assay was performed using a SimpleChIP Enzymatic Chromatin IP Kit (Cell Signaling Technology, Boston, MA) according to the manufacturer's instructions. Briefly, cells were cross-linked with $1 \%$ formaldehyde for 10 minutes at room temperature and then harvested and incubated on ice for 10 minutes in lysis buffer. Nuclei were pelleted and digested with micrococcal nuclease. Following sonication and centrifugation, sheared chromatin was incubated with anti-STAT3 or rabbit serum (negative control) overnight at $4^{\circ} \mathrm{C}$. Then, protein $\mathrm{G}$ beads were added, and the chromatin was incubated for 2 hours in rotation. Antibody-bound protein-DNA complexes were eluted and subjected to PCR analysis. The primers to amplify the human CD36 promoter were F': -201 and R: +1, which generate a 200bp product that covers the GAS binding site-93 to $-83 \mathrm{bp}$ upstream of CD36; F': +120 and R': +256, which generate a 136-bp product that covers the GAS binding site +187 to +196 bp upstream of CD36; F': +236 and R': +406, which generate a $170-b p$ product that covers the GAS binding site +363 to +374 bp upstream of $C D 36$; and $F^{\prime}:+355$ and R': +427 , which generate a 72 -bp product that covers the GAS binding site +382 to +391 .

\section{Electrophoretic mobility shift assay (EMSA)}

Non-denatured cellular nuclear extracts were prepared using a NE-PER extraction kit (Thermo Scientific Pierce, Rockford, IL). Nuclear protein extracts were incubated with biotin-labeled CD36 promoters' DNA probes in binding buffer for 30 minutes on ice. All probes which target GAS binding sites were synthesized by Sigma-Genosys (The Woodlands, TX). Following incubation, the samples were separated on a $5 \%$ polyacrylamide gel, transferred onto a nylon membrane, 
Table 1: Patient characteristics $(n=25)$

\begin{tabular}{lcc}
\hline Characteristic & Measure/Category & Overall \\
\hline Age, years & Median (range) & $58(40-78)$ \\
WBC $\times 10^{9} / \mathrm{L}$ & Median (range) & $27(7-55)$ \\
ALC $\times 10^{9} / \mathrm{L}$ & Median (range) & $22(1-48)$ \\
Rai stage & $(0,1-2 / 3-4)(\%)$ & $(95 / 5)$ \\
CD38 & $\leq 30 />30(\%)$ & $84 / 16(\%)$ \\
Zap-70 & Negative/Positive & $78 / 22(\%)$ \\
$\beta$ 3MM $(\mathbf{m g} / \mathbf{L})$ & $(</ \geq 4$ mg/L) & $95 / 5(\%)$ \\
IGHV mutation & $(\mathrm{M} / \mathrm{UM})$ & $78 / 22(\%)$ \\
FISH result, $\boldsymbol{n}$ & del17p/11q/T12/13q/Negative & $1 / 3 / 3 / 10 / 2$ \\
Karyotype, $\boldsymbol{n}$ & Diploid/Non-diploid/Complex/not done & $11 / 7 / 0 / 1$ \\
Survival status, $(\boldsymbol{n})$ & Alive/Dead & $19 / 0$ \\
\hline
\end{tabular}

WBC, white blood cell count; ALC, absolute lymphocyte count; $\beta 2 \mathrm{M}$, beta-2 microglobulin; M, mutated; UM, unmutated; FISH, fluorescence in situ hybridization.

and fixed on the membrane via ultraviolet cross-linking. The biotin-labeled probe was detected with strepavidinhorseradish peroxidase (Gel-Shift Kit; Panomics, Fremont, CA). The control consisted of 7-fold excess unlabeled cold probe. To test the effect of STAT3, anti-STAT3 antibodies (BD Bioscience) mouse IgG1 (BD Bioscience) were added with the nuclear extracts $[15,36]$.

\section{Transfection of MM-1 cells with CD36 gene promoter fragments and luciferase assay}

Four different CD36 promoter fragments, corresponding to the above described putative STAT3binding sites, were transfected into MM-1 cells by using electroporation. MM-1 cells were used because in these cells STAT3 phosphorylation is induced by extracellular signals such as IL-6. Each construct harbored a luciferase reporter gene and a CD36 promoter fragment that included at least $1 \gamma$-interferon activation sequence (GAS)-like elements. The luciferase activity of unstimulated or IL6-stimulated MM-1 cells was assessed 24 hours after transfection using a Dual-Luciferase Reporter Assay System (Promega) and a Sirius luminometer V3.1 (Berthold Detection Systems, Pforzheim, Germany). The luciferase activity of each of the human CD36 promoter constructs was determined by calculating the constructs' luciferase activity relative to the activity of the Renilla luciferase produced by the pRL-SV40 control vector.

\section{Generation of GFP-conjugated lentiviral STAT3 short hairpin RNA (shRNA) and infection of CLL cells}

293T cells were co-transfected with GFP-conjugated lentiviral STAT3 shRNA or a GFP-conjugated empty lentiviral vector and with packaging vectors (pCMV delta R8.2 and pMDG generously provided by Dr. Giorgio
Inghirami, (department of pathology, University of Torino, Italia) using the Superfect transfection reagent (QIAGEN Inc.) as previously described [15]. The 293T cell culture medium was replaced after 16 hours and collected after 48 hours. Then, the culture medium was filtered through a $45-\mu \mathrm{m}$ syringe filter to remove floating cells, the lentivirus was concentrated by filtration through an Amicon Ultra centrifugal filter device (EMD), and the concentrated supernatant was used to transfect CLL cells. CLL cells $\left(5 \times 10^{6} / \mathrm{ml}\right)$ were incubated in six-well plates (Becton, Dickinson and Company, Franklin Lakes, NJ) in $2 \mathrm{~mL}$ of Dulbecco's modified Eagle medium (DMEM) supplemented with $10 \%$ fetal bovine serum (FBS) and were transfected with $100 \mu$ l of viral supernatant. Polybrene $(10 \mathrm{ng} / \mathrm{mL})$ was added to the viral supernatant at a ratio of 1:1000 (v/v). Transfection efficiency was measured after 48 hours and ranged between $40 \%$ and $50 \%$ (calculated on the basis of the ratio of propidium iodidenegative and GFP-positive cells). These experiments were conducted using an upgraded FACSCalibur flow cytometer (Becton, Dickinson).

\section{Transfection of CLL cells with CD36 small interfering RNA (siRNA)}

Hundred $\mu \mathrm{M}$ human CD36-siRNA, $10 \mu \mathrm{l}$ FAMlabeled siRNA, targeting the human glyceraldehyde 3-phosphate dehydrogenase, or a scrambled control (Applied Biosystems, Foster City, CA) were added to $10 \mu \mathrm{l}$ siPORT NeoFX transfection reagent diluted in $50 \mu \mathrm{l}$ Opti-MEMI reduced serum medium (Thermo Fisher Scientific), and incubated at room temperature for 10 minutes. The transfection agents were incubated at room temperature with $1 \times 10^{7} \mathrm{CLL}$ cells suspended in 0.2 ml Opti-MEM I medium. After 1 hour of incubation, electroporation (Bio-Rad Laboratories) was performed and the cells were incubated in RPMI supplemented with $10 \%$ 
FBS for 24 hours. Transfection efficiency of the FAMconjugated siRNA was assessed on a FACSCalibur flow cytometer (Becton Dickinson Biosciences).

\section{Measurement of cellular $\mathrm{O}_{2}$ consumption}

Because FA metabolism increases $\mathrm{O}_{2}$ consumption, palmitic acid and oleic acid utilization was assessed by measuring the level of dissolved $\mathrm{O}_{2}\left(\mathrm{dO}_{2}\right)$ using the SevenGo pro Dissolved Oxygen Meter (Mettler Toledo, Worthington Columbus, $\mathrm{OH}$ ).

Preliminary experiments designed to test FA consumption that used palmitic acid or oleic acid dissolved in ethanol, determined that the $\mathrm{O}_{2}$ consumption with $80 \mathrm{mM}$ oleic acid or $80 \mathrm{mM}$ palmitic acid (both from Sigma-Aldrich) each is maximal therefore we used these concentrations in the following experiments. In each experiment we used CLL cells at a concentration of $2 \times 10^{6}$ cells $/ \mathrm{ml}$. The cells were incubated with a minimum essential medium (MEM) with Hank's salts and L-glutamine (Life Technologies, Carlsbad, CA) or with phosphate buffered saline medium (Invitrogen) for 48 to 72 hours in tightly sealed T25 tissue culture flasks (Corning, Tewksbury, MA ) at $37^{\circ} \mathrm{C}$ in the presence or absence of palmitic acid or oleic acid. The $\mathrm{O}_{2}$ meter probe was placed in the flask and the reading allowed stabilizing. Then, the dissolved $\mathrm{O}_{2}\left(\mathrm{dO}_{2}\right)$ level was recorded. The probe was cleaned before it was re-used. Measurements of $\mathrm{dO}_{2}$ were repeated at least three times for every data point. The $\mathrm{dO}_{2}$ of CLL cells transfected with CD36 siRNA, or incubated either with $2500 \mathrm{mg} /$ ml CD36-neutralizing antibodies (Abcam), with 100 $\mu \mathrm{M}$ of the LPL inhibitor orlistat, or $100 \mu \mathrm{M}$ of the CD36 inhibitor sulfosuccinimidyl oleate sodium (SSO) was assessed.

We used the Student $t$-test when one experimental condition was compared to non-treated (controls) and one-way ANOVA when 2 experimental conditions were compared to controls. Statistical analyses were performed using GraphPad version 5 (San Diego, California, USA).

\section{Annexin V/propidium iodide apoptosis assay}

The rate of cellular apoptosis was analyzed using double staining with a Cy5-conjugated annexin $\mathrm{V}$ kit and propidium iodide (PI; BD Biosciences) according to the manufacturer's instructions. Briefly, $1 \times 10^{6} \mathrm{CLL}$ cells were incubated for 24 hours in glucose-free MEM (Life Technologies) supplemented with 10\% FCS. After incubation the cells were washed once with phosphatebuffered saline and resuspended in $200 \mu \mathrm{L}$ binding buffer with $0.5 \mu \mathrm{g} / \mathrm{ml}$ annexin $\mathrm{V}-\mathrm{Cy} 5$ and $2 \mu \mathrm{g} / \mathrm{ml}$ propidium iodide (PI). After incubation for 10 minutes in the dark at room temperature, the samples were analyzed on a FACSCalibur flow cytometer (Becton Dickinson Biosciences). Cell viability was calculated as the percentage of annexin V-positive cells.

\section{CONFLICTS OF INTEREST}

All authors report no conflicts of interest.

\section{FINANCIAL SUPPORT}

This study was supported by the Cancer Center Support Grant from the NIH/NCI, P30 CA016672 grant.

\section{REFERENCES}

1. Bueso-Ramos CE, Ferrajoli A, Medeiros LJ, Keating MJ, Estrov Z. Aberrant morphology, proliferation, and apoptosis of B-cell chronic lymphocytic leukemia cells. Hematology. 2004; 9:279-86.

2. Chiorazzi N, Ferrarini M. Evolving view of the in-vivo kinetics of chronic lymphocytic leukemia B cells. Hematology Am Soc Hematol Educ Program. 2006; 2006: 273-8, 512.

3. Gibson SE, Leeman-Neill RJ, Jain S, Piao W, Cieply KM, Swerdlow SH. Proliferation centres of chronic lymphocytic leukaemia/small lymphocytic lymphoma have enhanced expression of MYC protein, which does not result from rearrangement or gain of the MYC gene. Br J Haematol. 2016; 175:173-5.

4. Rozovski U, Harris DM, Li P, Liu Z, Wu JY, Grgurevic S, Faderl S, Ferrajoli A, Wierda WG, Martinez M, Verstovsek S, Keating MJ, Estrov Z. At High Levels, Constitutively Activated STAT3 Induces Apoptosis of Chronic Lymphocytic Leukemia Cells. J Immunol. 2016; 196:4400-9.

5. Chiorazzi N. Cell proliferation and death: forgotten features of chronic lymphocytic leukemia B cells. Best Pract Res Clin Haematol. 2007; 20:399-413.

6. Neese RA, Misell LM, Turner S, Chu A, Kim J, Cesar D, Hoh R, Antelo F, Strawford A, McCune JM, Christiansen M, Hellerstein MK. Measurement in vivo of proliferation rates of slow turnover cells by $2 \mathrm{H} 2 \mathrm{O}$ labeling of the deoxyribose moiety of DNA. Proc Natl Acad Sci U S A. 2002; 99:15345-50.

7. McCaw L, Shi Y, Wang G, Li YJ, Spaner DE. Low Density Lipoproteins Amplify Cytokine-signaling in Chronic Lymphocytic Leukemia Cells. EBioMedicine. 2017; 15:24-35.

8. Rozovski U, Grgurevic S, Bueso-Ramos C, Harris DM, Li P, Liu Z, Wu JY, Jain P, Wierda W, Burger J, O'Brien S, Jain N, Ferrajoli A, et al. Aberrant LPL Expression, Driven by STAT3, Mediates Free Fatty Acid Metabolism in CLL Cells. Mol Cancer Res. 2015; 13:944-53.

9. Rozovski U, Hazan-Halevy I, Barzilai M, Keating MJ, Estrov Z. Metabolism pathways in chronic lymphocytic leukemia. Leuk Lymphoma. 2016; 57:758-65.

10. Martin CA, Longman E, Wooding C, Hoosdally SJ, Ali S, Aitman TJ, Gutmann DA, Freemont PS, Byrne B, Linton KJ. Cd36, a class B scavenger receptor, functions as a monomer to bind acetylated and oxidized low-density lipoproteins. Protein Sci. 2007; 16:2531-41. 
11. Coort SL, Willems J, Coumans WA, van der Vusse GJ, Bonen A, Glatz JF, Luiken JJ. Sulfo-N-succinimidyl esters of long chain fatty acids specifically inhibit fatty acid translocase (FAT/CD36)-mediated cellular fatty acid uptake. Mol Cell Biochem. 2002; 239:213-9.

12. Kerkhoff C, Sorg C, Tandon NN, Nacken W. Interaction of S100A8/S100A9-arachidonic acid complexes with the scavenger receptor CD36 may facilitate fatty acid uptake by endothelial cells. Biochemistry. 2001; 40:241-8.

13. Pohl J, Ring A, Korkmaz U, Ehehalt R, Stremmel W. FAT/ CD36-mediated long-chain fatty acid uptake in adipocytes requires plasma membrane rafts. Mol Biol Cell. 2005; 16:24-31.

14. Bilban M, Heintel D, Scharl T, Woelfel T, Auer MM, Porpaczy E, Kainz B, Krober A, Carey VJ, Shehata M, Zielinski C, Pickl W, Stilgenbauer S, et al. Deregulated expression of fat and muscle genes in B-cell chronic lymphocytic leukemia with high lipoprotein lipase expression. Leukemia. 2006; 20:1080-8.

15. Hazan-Halevy I, Harris D, Liu Z, Liu J, Li P, Chen X, Shanker S, Ferrajoli A, Keating MJ, Estrov Z. STAT3 is constitutively phosphorylated on serine 727 residues, binds DNA, and activates transcription in CLL cells. Blood. 2010; 115:2852-63.

16. Heinemeyer T, Wingender E, Reuter I, Hermjakob H, Kel AE, Kel OV, Ignatieva EV, Ananko EA, Podkolodnaya OA, Kolpakov FA, Podkolodny NL, Kolchanov NA. Databases on transcriptional regulation: TRANSFAC, TRRD and COMPEL. Nucleic Acids Res. 1998; 26:362-7.

17. Aaronson DS, Horvath CM. A road map for those who don't know JAK-STAT. Science. 2002; 296:1653-5.

18. Abumrad NA, el-Maghrabi MR, Amri EZ, Lopez E, Grimaldi PA. Cloning of a rat adipocyte membrane protein implicated in binding or transport of long-chain fatty acids that is induced during preadipocyte differentiation. Homology with human CD36. J Biol Chem. 1993; 268:17665-8.

19. Ibrahimi A, Sfeir Z, Magharaie H, Amri EZ, Grimaldi P, Abumrad NA. Expression of the CD36 homolog (FAT) in fibroblast cells: effects on fatty acid transport. Proc Natl Acad Sci U S A. 1996; 93:2646-51.

20. Frank DA, Mahajan S, Ritz J. B lymphocytes from patients with chronic lymphocytic leukemia contain signal transducer and activator of transcription (STAT) 1 and STAT3 constitutively phosphorylated on serine residues. J Clin Invest. 1997; 100:3140-8.

21. Rozovski U, Calin GA, Setoyama T, D'Abundo L, Harris DM, Li P, Liu Z, Grgurevic S, Ferrajoli A, Faderl S, Burger JA, O'Brien S, Wierda WG, et al. Signal transducer and activator of transcription (STAT)-3 regulates microRNA gene expression in chronic lymphocytic leukemia cells. Mol Cancer. 2013; 12:50.

22. Li P, Harris D, Liu Z, Rozovski U, Ferrajoli A, Wang Y, Bueso-Ramos C, Hazan-Halevy I, Grgurevic S, Wierda W,
Burger J, O'Brien S, Faderl S, et al. STAT3-activated GM-CSFRalpha translocates to the nucleus and protects CLL cells from apoptosis. Mol Cancer Res. 2014; 12:1267-82.

23. Li P, Harris D, Liu Z, Liu J, Keating M, Estrov Z. Stat3 activates the receptor tyrosine kinase like orphan receptor-1 gene in chronic lymphocytic leukemia cells. PLoS One. 2010; 5:e11859.

24. Demaria M, Camporeale A, Poli V. STAT3 and metabolism: how many ways to use a single molecule? Int J Cancer. 2014; 135:1997-2003.

25. Shulga N, Pastorino JG. GRIM-19-mediated translocation of STAT3 to mitochondria is necessary for TNF-induced necroptosis. J Cell Sci. 2012; 125:2995-3003.

26. Febbraio M, Abumrad NA, Hajjar DP, Sharma K, Cheng W, Pearce SF, Silverstein RL. A null mutation in murine CD36 reveals an important role in fatty acid and lipoprotein metabolism. J Biol Chem. 1999; 274:19055-62.

27. Nath A, Li I, Roberts LR, Chan C. Elevated free fatty acid uptake via CD36 promotes epithelial-mesenchymal transition in hepatocellular carcinoma. Sci Rep. 2015; 5:14752.

28. Pascual G, Avgustinova A, Mejetta S, Martin M, Castellanos A, Attolini CS, Berenguer A, Prats N, Toll A, Hueto JA, Bescos C, Di Croce L, Benitah SA. Targeting metastasisinitiating cells through the fatty acid receptor CD36. Nature. 2017; 541:41-5.

29. Kuda O, Pietka TA, Demianova Z, Kudova E, Cvacka J, Kopecky J, Abumrad NA. Sulfo-N-succinimidyl oleate (SSO) inhibits fatty acid uptake and signaling for intracellular calcium via binding CD36 lysine 164: SSO also inhibits oxidized low density lipoprotein uptake by macrophages. J Biol Chem. 2013; 288:15547-55.

30. Kusaka Y, Tanaka T, Okamoto F, Terasaki F, Matsunaga Y, Miyazaki H, Kawamura K. Effect of sulfo-N-succinimidyl palmitate on the rat heart: myocardial long-chain fatty acid uptake and cardiac hypertrophy. J Mol Cell Cardiol. 1995; 27:1605-12.

31. Glatz JF, Angin Y, Steinbusch LK, Schwenk RW, Luiken JJ. CD36 as a target to prevent cardiac lipotoxicity and insulin resistance. Prostaglandins Leukot Essent Fatty Acids. 2013; 88:71-7.

32. Mansor LS, Sousa Fialho MDL, Yea G, Coumans WA, West JA, Kerr M, Carr CA, Luiken JJFP, Glatz JFC, Evans RD, Griffin JL, Tyler DJ, Clarke K, Heather LC. Inhibition of sarcolemmal FAT/CD36 by sulfo-N-succinimidyl oleate rapidly corrects metabolism and restores function in the diabetic heart following hypoxia/reoxygenation. Cardiovasc Res. 2017; 113:737-48.

33. Greenwalt DE, Scheck SH, Rhinehart-Jones T. Heart CD36 expression is increased in murine models of diabetes and in mice fed a high fat diet. J Clin Invest. 1995; 96:1382-8.

34. Ferrajoli A, Faderl S, Van Q, Koch P, Harris D, Liu Z, Hazan-Halevy I, Wang Y, Kantarjian HM, Priebe W, Estrov Z. WP1066 disrupts Janus kinase-2 and induces caspase-dependent apoptosis in acute myelogenous leukemia cells. Cancer Res. 2007; 67:11291-9. 
35. Harris DM, Hazan-Haley I, Coombes K, Bueso-Ramos C, Liu J, Liu Z, Li P, Ravoori M, Abruzzo L, Han L, Singh S, Sun M, Kundra V, et al. Transformation of human mesenchymal cells and skin fibroblasts into hematopoietic cells. PLoS One. 2011; 6:e21250.
36. Liu Z, Hazan-Halevy I, Harris DM, Li P, Ferrajoli A, Faderl S, Keating MJ, Estrov Z. STAT-3 activates NF-kappaB in chronic lymphocytic leukemia cells. Mol Cancer Res. 2011; 9:507-15. 